\title{
Primary Laryngeal Tuberculosis
}

\author{
Madiha Mahfoudhi ${ }^{*}$, Khaled Khammassi², Amel Gaieb Battikh'1, Mamia Ben Salah², \\ Sami Turki ${ }^{1}$ \\ ${ }^{1}$ Department of Internal Medicine, A. Charles Nicolle Hospital, Tunis, Tunisia \\ ${ }^{2}$ Department of Oto-Rhino-Laryngology, Charles Nicolle Hospital, Tunis, Tunisia \\ Email: ${ }^{*}$ madiha mahfoudhi@yahoo.fr
}

Received 18 December 2014; accepted 7 April 2015; published 14 April 2015

Copyright (C) 2015 by authors and Scientific Research Publishing Inc.

This work is licensed under the Creative Commons Attribution International License (CC BY).

http://creativecommons.org/licenses/by/4.0/

(c) (i) Open Access

\section{Abstract}

Primary laryngeal tuberculosis is rare. It can manifest as a malignancy delaying the diagnosis and the therapeutic management. We report the case of a 34-year-old immunocompetent man whose plaints were fever, sweat and dysphonia. There were no adenopathy, nor hepatosplenomegaly. Several blood cultures were negative. Laboratory studies showed an inflammatory syndrome. In addition, the bacteriological examinations of sputum and urine in search of Mycobacterium tuberculosis were negative. Laryngoscopy confirmed the presence of a thickness of the right vocal cord and an ulcer in the hemilarynx. The histological examination revealed tuberculoid granulomas suggestive of tuberculosis. The culture from vocal cord tissue for Mycobacterium tuberculosis was positive. No other focus of tuberculosis was found on X-ray and computed tomography. Treatment by a combination of isoniazid, rifampicin, pyrazinamide induced a total resolution of symptoms.

\section{Keywords}

Larynx, Tuberculosis, Dysphonia

\section{Introduction}

The tuberculosis is an infection due to Mycobacterium tuberculosis. The pulmonary form is the most frequent.

Tuberculosis can manifest as a latent and chronic form leading to irreducible tissue decay resistant to any type of treatment.

The control of tuberculosis vaccine permitted the decease of the prevalence of the incidence of this disease. However, some areas are still considered endemic zones in which the tuberculosis diagnosis should be considered. Hence, clinical features of tuberculosis have been changed because of the antituberculous drugs availability and various immunosuppressive conditions increase.

\footnotetext{
*Corresponding author.
} 
These conditions have favored the emergence of extrapulmonary forms of tuberculosis implicating rachis, meninges, hypophysis, thyroid, digestive system, larynx...

When laryngeal manifestations are associated to pulmonary signs, tuberculosis diagnosis is easy. However, primary laryngeal tuberculosis is rare and may manifest as a tumor inducing a delayed diagnosis time [1] [2]. Clinical and endoscopic feature have no specificity in case of primary laryngeal tuberculosis. Only typical histological results associating granulomas and caseous necrosis or bacteriological findings allow tuberculosis origin of laryngeal symptomatology.

The aim of this study is to demonstrate the diagnosis difficulty of extrapulmonary tuberculosis; as illustrate our case who presents laryngeal tuberculosis mimicking a tumoral origin.

\section{Case Report}

A 34-year-old immunocompetent and non smoking man presented a prolonged fever, sweat and recent weight loss evolving from one month. The interrogatory didn't find a personal antecedent of tuberculosis, or a tuberculosis case in his entourage. He was well vaccinated by BCG vaccine and had not received immusuppressive treatment. The physical examination revealed a fever of $39^{\circ} \mathrm{C}$ and a dysphonia. There were no adenopathy, nor hepatosplenomegaly. The cardiac, pulmonary, skinny, neurological and osteoarticular examinations were normal.

Several blood cultures performed in emergency were negative.

Laboratory studies objectified an inflammatory syndrome. He had an inflammatory microcytic hypochromic anemia (hemoglobin: $11.5 \mathrm{~g} / \mathrm{dl}$ ), a white blood cells count of $9500 / \mathrm{mm}^{3}$ and a normal platelets count of $470,000 / \mathrm{mm}^{3}$. There were no biological sign of malabsorption. The renal and hepatic functions were normal. In addition, the bacteriological examinations of sputum and urine in search of Mycobacterium tuberculosis were negative.

Besides, the immunological (ANA, ANCA) investigations were negative. The dosage of beta 2 microglobulin was in limit of the normal.

Laryngoscopy confirmed a thickness of the right vocal cord which was erythematous and a deep ulcer measuring $15 \mathrm{~mm}$ in the right hemilarynx. There was no polyp or vegetation. No paralysis or paresis of the vocal cords was mentioned.

The vocal cord and ulcer histological examination revealed inflammatory infiltrates organized in granulomas formed essentially of lymphocytes, epitheloid and giant cells, fibrosis and caseous necrosis, suggestive of tuberculosis and the ziehl-neelsen staining showed acid-alcohol resistant bacilli. The culture from vocal cord tissue for Mycobacterium tuberculosis was positive. No other focus of tuberculosis was found on X-ray and computed tomography.

Treatment with a combination of isoniazid, rifampicin, pyrazinamide, and ethambutol for two months and the association isoniazid and rifampicin for ten months induced a total resolution of clinical, biological and endoscopic signs.

\section{Discussion}

Isolated laryngeal tuberculosis in an immunocompetent patient is rare. Indeed, laryngeal tuberculosis is always associated to pulmonary location and occurs generally in patients without BCG vaccination or in cases of immune deficiency [1] [2].

Primary laryngeal tuberculosis can manifest as chronic hoarseness or a dysphonia evolving in a febrile context.

Fsadni, P. et al. reported the case of an 85-year-old woman who presented with a 6-month history of persistent hoarseness in whom the definitive diagnosis was a primary laryngeal tuberculosis confirmed by histological results [2].

El Ayoubi, F. et al. studied ten cases of primary laryngeal tuberculosis. In this series, the patients were male smokers and drinkers, while our case had no particular antecedent. They had no specific clinical and endoscopic simulating those of laryngeal cancer. Only bacteriological and histological examinations made the diagnosis [1].

A malignancy, a lymphoma or a vasculitis may be considered the essential differential diagnosis. Endoscopic findings can be exophytic or ulcerative. The series study of Hasibi, M. et al. revealed that macroscopic aspects of laryngeal lesions were exophytic in 11 cases and ulcerative in 14 cases [3].

The laryngeal lesion is chronic, persistent and non-healing despite of a well conducted symptomatic treatment. 
The first clinical diagnosis was malignancy or nonspecific inflammation in many published cases [3]-[5].

El Kettani, N. E. et al. reported a case of a 36-year-old man suffering from dysphonia and dysphagia. Laryngoscopy showed a lesion of the left vocal cord and CT scan found focal irregular thickening of the posterior laryngeal wall evoking a laryngeal carcinoma [6].

Thus, laryngeal tuberculosis is initially diagnosed as carcinoma in several circumstances [7].

Positive tuberculin skin test and quantiferon gold are in favor of tuberculosis lesions. The ziehl-neelsen staining showing acid-alcohol resistant bacilli and positive specific culture in research of Mycobacterium tuberculosis are good arguments to retain the tuberculous origin.

The histopathology results can be typical as presented in our case (chronic granulomatous inflammation with caseous necrosis and langhans-type giant cells) confirming the diagnosis or atypical (chronic granulomatous inflammation or chronic inflammation without necrosis) delaying the diagnosis [3] [5].

Hasibi, M. presented 19 cases of laryngeal tuberculosis with typical tuberculosis histopathology (granulomas with caseous necrosis and giant cells); whereas 6 patients had atypical histopathology (granulomatous or chronic inflammation without necrosis). The clinical symptoms lasted from 2 to 12 months before diagnosis confirmation. This diagnosis delay can worsen the laryngeal prognosis [3].

The primary laryngeal tuberculosis should be suspected in endemic countries even if the clinical and endoscopic features are atypical [3] [4]. A lateness of diagnostic delay can cause extensive laryngeal tissue damage and spread of disease.

Only an early diagnosis and specific treatment allow the resolution of all symptoms.

The response to anti-tuberculosis therapy was desirable in our case and in the most publications [3] [6].

Once diagnosis confirmed, antituberculosis chemotherapy administered in ten patients for a minimum duration of 6 months induced excellent short-term and long-term results [1]. This duration was prolonged in our case to twelve months to avoid laryngeal irreducible damagement.

\section{Conclusion}

It's important to consider the tuberculosis as a disease which presents with variable clinical features and uncommon endoscopic findings, but for which medical treatment is very effective.

\section{Disclosure Statement}

The authors have nothing to disclose.

\section{References}

[1] El Ayoubi, F., Chariba, I., El Ayoubi, A., Chariba, S. and Essakalli, L. (2014) Primary Tuberculosis of the Larynx. European Annals of Otorhinolaryngology, Head and Neck Diseases, 131, 361-364. http://dx.doi.org/10.1016/j.anorl.2013.10.005

[2] Fsadni, P., Fsadni, C. and Caruana Montaldo, B. (2014) Primary Laryngeal Tuberculosis: An Unusual Cause of Hoarseness. Ear, Nose \& Throat Journal, 93, E15-E17.

[3] Hasibi, M., Yazdani, N., Asadollahi, M., Sharafi, M. and Dehghan Manshadi, S.A. (2013) Clinical Features of Laryngeal Tuberculosis in Iran. Acta Medica Iranica, 51, 638-641.

[4] Cruz, S., Ribeiro, A., Trigueiros, N., Rodrigues, E. and Rodrigues, M. (2014) Laryngeal Tuberculosis: A Diagnosis Not to Be Overlooked. European Annals of Otorhinolaryngology, Head and Neck Diseases, 131, 325-326. http://dx.doi.org/10.1016/j.anorl.2013.11.011

[5] Benwill, J.L. and Sarria, J.C. (2014) Laryngeal Tuberculosis in the United States of America: A Forgotten Disease. Scandinavian Journal of Infectious Diseases, 46, 241-249. http://dx.doi.org/10.3109/00365548.2013.877157

[6] El Kettani, N.E., El Hassani, M., Chakir, N. and Jiddane, M. (2010) Primary Laryngeal Tuberculosis Mimicking Laryngeal Carcinoma: CT Scan Features. Indian Journal of Radiology and Imaging, 20, 11-12. http://dx.doi.org/10.4103/0971-3026.59745

[7] Baxi, S. and Jha, S. (2010) Primary Laryngeal Tuberculosis-A Rare Entity. Journal of the Indian Medical Association, 108, 178-179.

[8] Levian, M., Chapman, A. and Gupta, R. (2014) Laryngeal Tuberculosis: Use of Videostroboscopy in Diagnosis. Ear, Nose \& Throat Journal, 93, 58. 